\title{
EDITORIAL
}

\section{A few ideas on training in radiology}

\section{C de Vries, MMed $(\operatorname{Rad} D)$}

Department of Radiology, University of the Free State
There has been a lot of discussion around the problems facing radiology registrars in South Africa. Some of these problems include the lack of consultant coverage in the academic setting, old equipment in training hospitals and not enough time spent on official teaching and tutoring as a result of the heavy work load faced by the radiology departments in the public sector. This prompts the questions - is our radiology training on a par with the rest of the world and does the training reflect these problems faced by academic radiology departments and the registrars they train? The answer is a very definite 'No'. By working around our problems we train world-class radiologists. New equipment has made an impact and registrars in radiology now have access to MRI on a daily basis, whereas before the installation of the equipment, registrars were only occasionally exposed to MRI from the private sector.

\section{New solutions}

The theoretical part of the registrar training could be addressed by a modular 4-year curriculum. This modular curriculum would be based on a sound knowledge of radiological anatomy, radiation physics, MRI physics and applied physiology which would then be assessed by the College of Radiology, or the university, during the primary exam. This 4-year approach divides the essential knowledge into modules, spread over a 5-year period in such a way that it can be entered into at any stage. At the end of 4 years the registrar will have been exposed to all the essential theory of radiology, irrespective of where or when he/she entered into the programme.

\section{Uniformity and co-operation}

The idea is to get all the training institutions on board and have a unified theoretical curriculum. Training and lectures can then be shared by all the participating universities. This can quite easily be achieved by making use of teleconferencing to facilitate a combined meeting, during which presented lectures can be interactively viewed and heard by the participating universities. This has recently been put into practice and a very successful teleconference meeting was held involving the universities of Bloemfontein, Pretoria and Stellenbosch (See 'Specialty corner' in this issue for current events).

\section{Glass half-full}

The problem regarding the high case loads can actually be viewed as an advantage to the training registrar. To become a radiologist you have to do radiology and the more you do, the more experience you will gain and the better you will become at your chosen specialty.

\section{The test: the 'real world'}

My question to South African-trained radiologists working in the UK, Australia and elsewhere in the world is this — was your training really that bad?

\section{Coert de Vries}

Guest Editor 